Wei-Erh Cheng Chuen-Ming Shih Liang-Wen Hang Kuen-Yuh Wu Hsin-Ling Yang Wu-Huei Hsu Te-Chun Hsia

\section{Urinary biomarker of oxidative stress correlating with outcome in critically septic patients}

Published online: 10 July 2007

(C) Springer-Verlag 2007

The online version of the original can be found at http://dx.doi.org/10.1007/s00134-007-0715-y.

W.-E. Cheng · C.-M. Shih · L.-W. Hang · W.-H. Hsu •

T.-C. Hsia (®)

China Medical University Hospital, Division of Pulmonary and

Critical Care Medicine, Department of Internal Medicine,

2 Yuh-Der Rd., 404 Taichung, Taiwan

e-mail: derrick.hsia@msa.hinet.net

Tel.: +886-4-22052121

Fax: +886-4-22038883

\section{K.-Y. Wu}

National Health Research Institute, Division of Environmental

Health and Occupational Medicine,

35 Keyan Road, 350 Miaoli County, Zhunan Town, Taiwan

H.-L. Yang

China Medical University, Department of Nutrition,

91 Hsueh-Shih Road, 404 Taichung, Taiwan

\section{Intensive Care Med (2007)}

DOI 10.1007/s00134-007-0715-y

Owing to an error in production, the names of the first two authors appeared in the wrong order. This mistake is corrected here.

Wei-Erh Cheng

Chuen-Ming Shih

Liang-Wen Hang

Kuen-Yuh Wu

Hsin-Ling Yang

Wu-Huei Hsu

Te-Chun Hsia 